\title{
A Cochlear Implant System with Infra-red Telemetry for Behaving Animals
}

\author{
Soon Kwan An ${ }^{1,3}$, Choong Jae Lee ${ }^{1,3}$, Kyoung Min Byun ${ }^{1,3}$, Sang Beom Jun ${ }^{1,3}$, Se Ik Park ${ }^{1,3}$, Min Hyun. Park ${ }^{2,3}$, \\ Seung $\mathrm{Ha} \mathrm{Oh}^{2,3}$, Sung June $\mathrm{Kim}^{1,3}$ \\ School of Electrical Engineering and Computer Science, Seoul National University ${ }^{1}$ \\ Department of Otolaryngology, Seoul National University College of Medicine ${ }^{2}$ \\ Nano Bioelectronics and Systems ERC, Seoul National University, Seoul, Korea ${ }^{3}$
}

\begin{abstract}
A new cochlear implant system for animal experiments is proposed. The system uses a DSP-based sound processor to implement the six-channel continuous interleaved sampling (CIS) speech processing strategy. An infra-red telemetry is designed to remotely connect the power-demanding sound processor to receiver/stimulator attached on the animal. This enables the animal to move freely. The receiver/stimulator consumes about $40 \mathrm{~mW}$, thus can operates for about $200 \mathrm{hours}$ without changing battery.
\end{abstract}

\section{INTRODUCTION}

Cochlear implant system is one of the most advanced neural prosthetic devices. Studies focused on the physiological response properties of auditory neurons to the cochlear electro-stimulation and central auditory plasticity by long-term cochlear implantation have been performed $[1,2,3]$. These studies need many animal experiments using some kinds of animal cochlear implant systems. In early animal experiments, simple electrical cochlear stimulator with fixed stimulation parameters or simple single channel human cochlear implant system was used $[1,2]$. These systems were simple and light-weighted but could not provide sound stimulations similar to natural and complex environmental sounds. Alternatively, commercial multi-channel human cochlear implant systems can be adapted [3]. These systems are based on based on commercial digital signal processing (DSP) technologies and implement multi-channel coding strategies, thus providing complex electrical stimuli according to the natural environmental sounds. These are too large and heavy for animals to move freely with them and need frequent recharging of batteries because of the high power consumption of the DSPs.

In this paper, we present a new cochlear implant system for freely moving animals. Our system also employed DSP but it is located on the experimental cage where external power is available. The animal carries a light-weighted package only which communicates with the sound processor on the cage through an infra-red telemetry, thus freeing the animal from the need to carry a heavy battery.

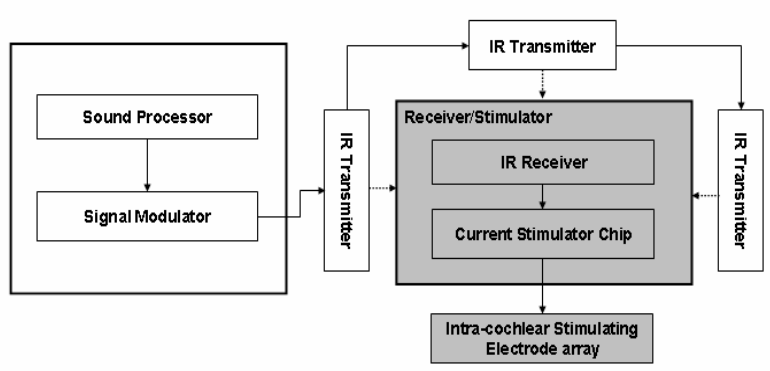

Figure 1. System block diagram.

\section{METHODS}

\section{A. System Structure}

Figure 1 shows the system structure. The system consists of two main parts - the external parts that is located on the experimental cage, and the internal parts that is carried by animals. The external parts are composed of a sound processor, a signal modulator and infra-red transmitters. They need much power because all the signal processing and modulation are performed in these parts. A receiver/stimulator and an intra-cochlear stimulating electrode array belong to the internal parts. Receiving the processed data from the external part, they decode them and generate current stimulation pulses.

\section{B. Sound Processor}

The six channel CIS speech processing strategy is implemented by the sound processor that consists of a microphone, a pre-amplifier, a band-pass filter and digital signal processing circuitry. First, acoustic sounds are converted into electrical signals by a microphone, then the electric signals are amplified, filtered and fed into the DSP circuitry. DSP circuitry performs a fast Fourier transform (FFT) to analyze sounds. Using digitally programmable bandpass filters, this sound processor extracts power spectrum and calculate its energy to encode the amplitude of current pulses for each channels sequentially. 


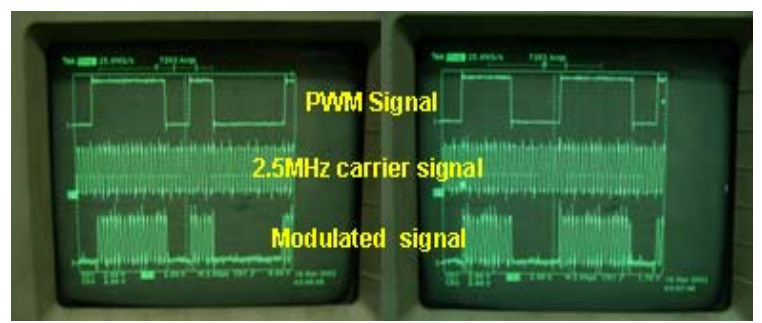

Figure 2. Signal modulation for IR transmission.

\section{Signal Modulator}

The resulting digital data of the sound processor are pulse width modulated (PWM) signals. These PWM coded signals need to be modulated to transmit using infra-red transmitters. The signal modulator converts these PWM signals into numbers of pulses to transmit these signals to the internal receiver/stimulator (figure 2).

\section{Infra-red Transmitters}

To transmit stimulation control signal, several infra-red transmitters are used. An IR transmitter used in our system is compatible with infra-red Data Association (IrDA) standard and be able to deliver data with rate up to 4Mbps. There are limitations in its transmission distance and transmission angle that are to be about 1.2 meters and about \pm 35 degrees. According to the position of animals, data transmission failure may occur by missing the alignment between an infra-red transmitter and an receiver. Because most of the experiments are carried out with the animals in a cage, one is more likely to face with the transmission angle problem than with the distance problem. In our system, this problem is solved by using multiple infra-red transmitters attached on many parts around the cage.

\section{E. Receiver/Stimulator}

The receiver/stimulator consists of an infra-red receiver and a current stimulator. Transmitted stimulation control data are received and demodulated by an infra-red receiver. Then the current stimulator decodes received data, and according to them, generates biphasic current pulses that are to be delivered sequentially to the six intra-cochlear stimulating electrode sites to stimulate spiral ganglia of animals. The current simulator was designed to provide current ranging up to $2 \mathrm{~mA}$ in biphasic pulses.

\section{F. Intra-cochlear Stimulating Electrode Array (Figure 3)}

A 6-channel intra-cochlear stimulating electrode array especially for cat - are designed and fabricated with slilconeelastomer molding technology. The size and insertion depth of the electrode array are designed according to the internal volume of cat scala-tympani.

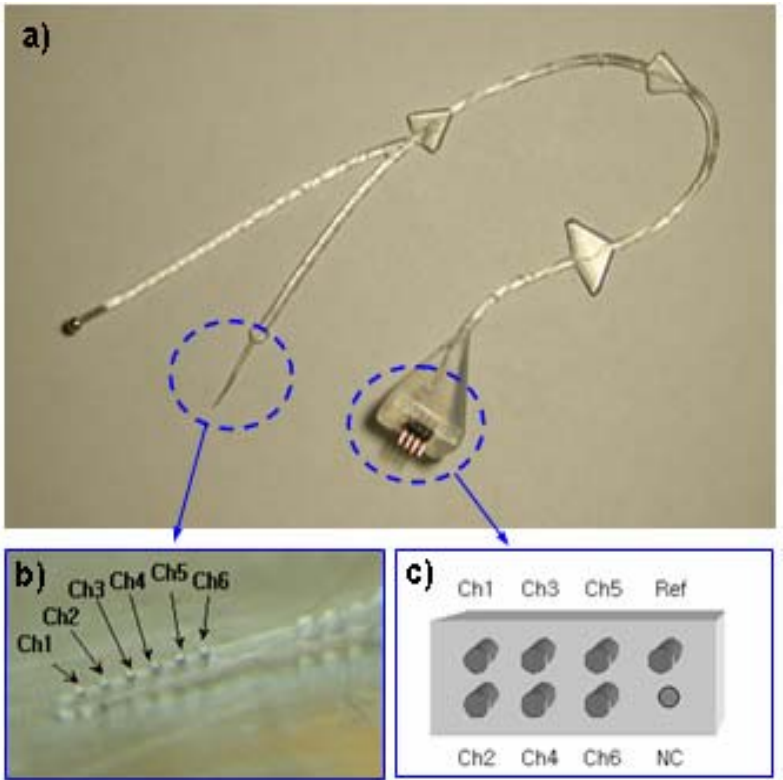

Figure 3. (a) Intra-cochlear stimulating electrode array. (b) six electrode ball contacts, (c) Pin configurations.

The electrode array consists of six channel stimulating electrodes, a reference electrode, a connector for interface with a receiver/stimulator. Six teflon-coated, platinum-iridium wires (90\%:10\%), embedded in a silicone elastomer carrier, and each of which ended in a ball contact. The electrode impedance was measured to be about 4 kohms(@1kHz).

Three triangular structures are added in the electrode carrier to help the electrode array be in stable position. They are positioned under the skin of the animal and by suturing these structures with the tissues, the electrode array can be tightly fixed inside animal body. The one structure that has different shape with the others is designed to prevent the electrode array from pulling out by external forces.

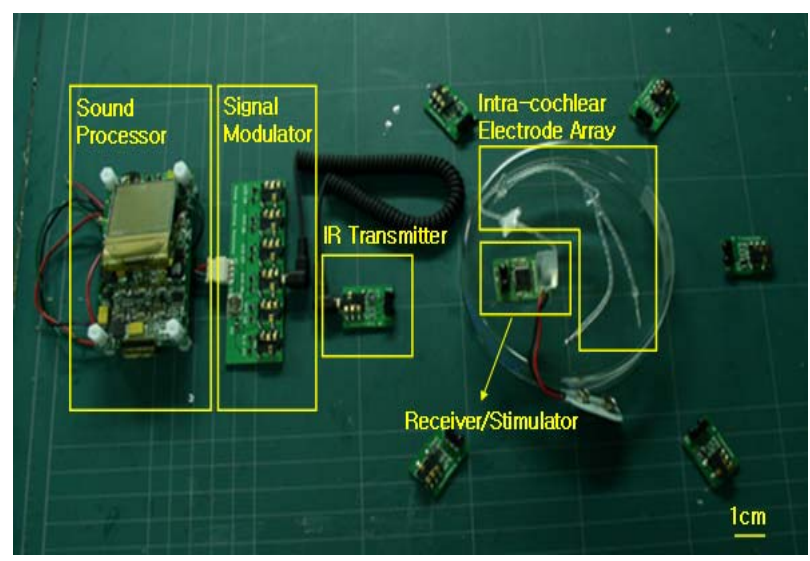

Figure 4. Cochlear implant system for animal experiments. 

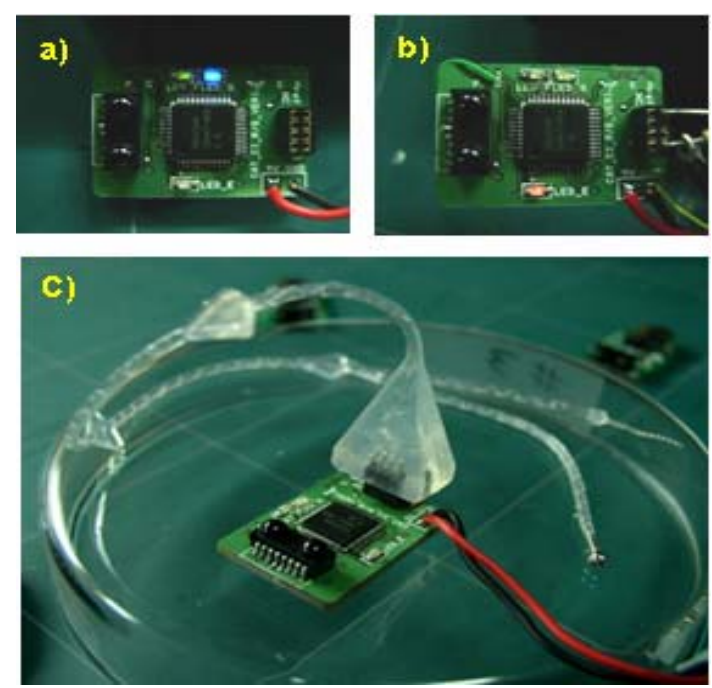

Figure 5. The internal part.

Transmission feedback with LEDs, a) Transmission status is okay, b) failed,

c) interfacing between a receiver/stimulator and intra-cochlear electrode array.

\section{RESULTS}

Figure 4 shows the implemented cochlear implant system. The external part that have large volume and consume much power is equipped out of the experimental cage, and powered by external power supply. A signal modulator can be connected with up to six infra-red transmitters and the number of infra-red transmitters can be expanded by using additional signal modulators.

The receiver/stimulator is $17 \mathrm{~mm} \times 25 \mathrm{~mm}$ in size and weigh only about 3.8 grams. Implanted intra-cochlear stimulating electrode array is connected with a receiver/stimulator by a small connector and there are light-emitting diodes to monitoring the transmission status (figure 5).

Functionality and power consumption of the system were checked. Six infra-red transmitters are connected with a signal modulator, and to observe the exact biphasic current pulses, one-channel of the intra-cochlear electrode array is replaced with $10 \mathrm{kohm}$ resistor load. As in figure 6, implemented system works well and generates well-balanced biphasic current pulses. The total power consumption of the internal part is about $40 \mathrm{~mW}$.

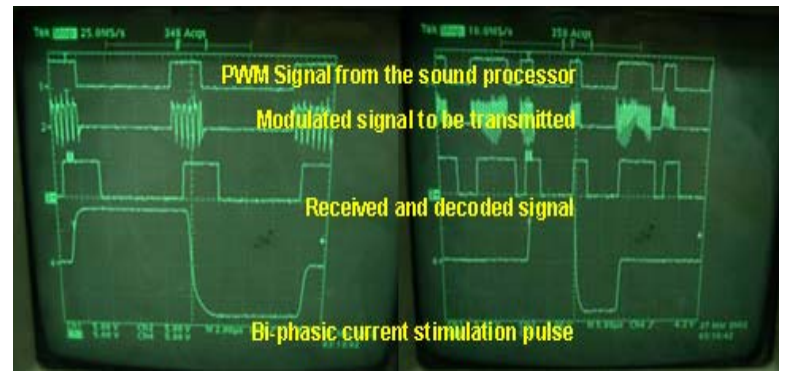

Figure 6. Results of the functionality test
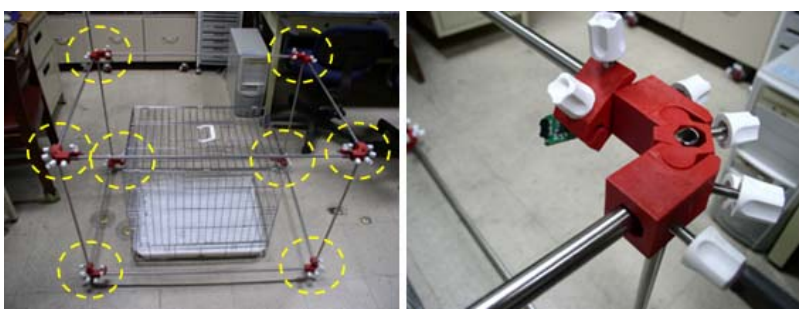

Figure 7. An experimental cage with multiple IR transmitters.
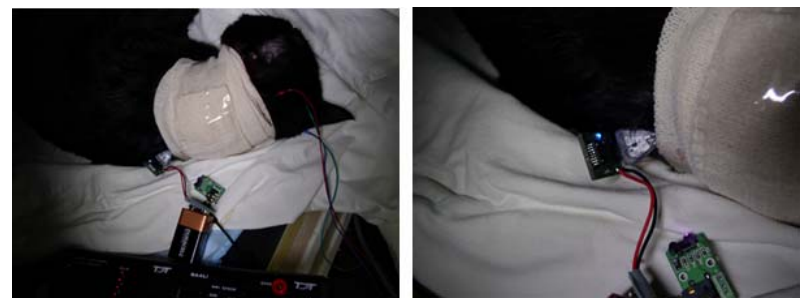

Figure 8. A Cat with the receiver/stimulator and the electrode array

\section{SUMMARY AND DISCUSSION}

A noble cochlear implant system for animal experiments was developed and its functionality and power consumption was checked. Using infra-red telemetry, the receiver /stimulator can be separated from external sound processor. This scheme enables animals to reduce their loads and to move more freely while providing stimuli similar to the natural environmental sound that needs complex and muchpower consuming signal processing. Transmission failure that may occur by misalignment of transmitter/receiver can be solved by employing multiple infra-red transmitters located on many part of the experimental cage (figure 7).

The receiver/stimulator is only $17 \mathrm{~mm} \times 25 \mathrm{~mm}$ in size and weigh only about 3.8 grams. Total power dissipation of the internal part is about $40 \mathrm{~mW}$, so with $9 \mathrm{~V}, 1000 \mathrm{mAh}$ lithium $\mathrm{MnO}_{2}$ battery, it can operate for about 200 hours continuously.

\section{ACKNOWLEDGEMENT}

This work was supported by Korea Science and Engineering Foundation (KOSEF) through Nano Bioelectronics and Systems Research Center (NBS-ERC) in Seoul National University.

\section{REFERENCES}

[1] Rainer Klinke, Andrej Kral, Jochen Tillein, Rainer Hartmann, "Recruitment of the Auditory Cortex in Congenitally Deaf Cats by LongTerm cochlear Electrostimulation," Science, vol. 285, pp. 1729-1733, September 1999.

[2] Andrej Kral, Rainer Hartmann, Dariusch Mortazavi, Rainer Klinke, "Spatial resolution of cochlear implant : the electrocal field and excitation of auditory afferents," Hearing Research, vol. 121, pp. 11-28, 1998.

[3] Erika A. Kretzmer, Noah E. Meltzer, Charks-Andre Haenggeli, David K. Ryugo, "An Animal Model for Cochlear Implants," Arch Otolaryngol Head Neck Surg., vol. 130, pp. 499-508, May 2004. 